\title{
Kinetics of Ordering in Fe-Al Alloys
}

\author{
By Kensuke Oki*, Masayuki Hasaka** and Tetsuo Eguchi*
}

\begin{abstract}
The kinetics of ordering in $\mathrm{Fe}-\mathrm{Al}$ alloys with about 25 at \% $\mathrm{Al}$ is investigated both theoretically and experimentally. The kinetic equations which express the time variation of the degrees of order in $\mathrm{B} 2$ and $\mathrm{DO}_{3}$ structures are obtained by takjng into account the direct interchange of atoms between the first and the second nearest neighbors. The experimental results of X-ray diffraction for these alloys on isothermal annealing or slow cooling are reproduced satisfactorily by theoretical rate equations, and the characteristics of the ordering in the $\mathrm{Fe}-\mathrm{Al}$ system is investigated. The ordering in $\mathrm{DO}_{3}$ type is influenced substantially by the atomic interchange between the second nearest neighbors. The $\mathrm{B} 2$ type ordering takes place so rapidly that the quenching from a disordered phase does not prevent the ordering. An oscillatory change of the degree of order in the $\mathrm{B} 2$ structure is expected from the calculation in the isothermal annealing.
\end{abstract}

(Received August 27, 1973)

\section{Introduction}

Kinetic studies of ordered alloys have been actively performed both experimentally and theoretically. They are useful in interpreting the variation of physical quantities of the alloys under various heat treatments. In almost all circumstances the arrangement of atoms in an alloy is in a non-equilibrium state, but it tends towards the equilibrium with a finite relaxation time, which, in return, depends upon the internal state of the alloy. Thus it is necessary for us to make a kinetic consideration in the analysis of experimental data on ordering or clustering of an alloy.

The theoretical investigation for this purpose has been done mainly with $\mathrm{L}_{0}$ and $\mathrm{L}_{2}$ superstructures $^{(1) \sim(7)}$. With regard to the alloy systems, however, which have two types of superstructures of B2 and $\mathrm{DO}_{3}$ types, such as $\mathrm{Fe}-\mathrm{Al}$ system with about 25 at $\%$ $\mathrm{Al}$, no theoretical investigations dealing with the ordering kinetics have been reported in the literature. On the other hand a number of kinetic studies with the $\mathrm{Fe}-\mathrm{Al}$ alloys have been carried out experimentally $^{(8) \sim(13)}$, but no adequate analysis has been performed because of the lack of the rate equations describing the change of atomic arrangement in these alloys.

In the present paper a set of kinetic equations for the ordering in $\mathrm{Fe}-\mathrm{Al}$ system with two types of superstructures $\mathrm{B} 2$ and $\mathrm{DO}_{3}$ are obtained, and the experimental results on the process of ordering in the alloys are interpreted with the aid of the rate equations. Considering the direct interchange of atoms between the first and the second nearest neighbors, we derive the kinetic equations which express the time variation of the degrees of order in $\mathrm{B} 2$ and $\mathrm{DO}_{3}$ superstructures. The simultaneous differential equations thus obtained are linearized by a Taylor expansion around the equilibrium degrees of order, and are applied for the analysis of the main kinetic features of ordering which have been observed in various ex-

* Department of Iron and Steel Metallurgy, Faculty of Engineering, Kyushu University, Fukuoka, Japan.

** Graduate School, Kyushu University, Fukuoka, Japan. Trans. JIM perimental investigations. The variation of the degrees of order in the $\mathrm{Fe}-\mathrm{Al}$ either quenched from various temperatures or annealed isothermally is obtained by means of X-ray diffraction, and the experimental data are analyzed satisfactorily with our rate equations.

\section{Basic Equations}

\section{Derivation of basic equations}

It is known that the $\mathrm{Fe}-\mathrm{Al}$ alloys with about 25 at \% Al have two types of superstructures, namely B2 and $\mathrm{DO}_{3}{ }^{(14)}$. We consider a perfect crystal of an alloy of $\mathrm{Fe}$ and $\mathrm{Al}$ atoms with the composition $\mathrm{Fe}_{3-\varepsilon} \mathrm{Al}_{1+\varepsilon}$, where these atoms are distributed in the lattice with a unit cell illustrated in Fig. 1. The lattice sites are usually classified into three kinds, $\alpha, \beta$ and $\gamma$ sites. The numbers of $\mathrm{Fe}$ and $\mathrm{Al}$ atoms on each kind of lattice sites can be expressed in terms of two parameters $x$ and $y$, which represent the degrees of order in $\mathrm{DO}_{3}$ and $\mathrm{B} 2$ superstructures, respectively. Their definition is identical with those of our previous work ${ }^{(15)}$ as shown in Table 1.

In the $\mathrm{DO}_{3}$ state, which is stable below a certain temperature $T_{x}, x$ and $y$ do not vanish, but in the $\mathbf{B} 2$ state within the temperature range from $T_{x}$ up to $T_{y}$, $x$ vanishes but $y$ does not, whereas in the disordered state above $T_{y}$ both $x$ and $y$ vanish. If the values of $x$ and $y$ happen to differ from their equilibrium values,
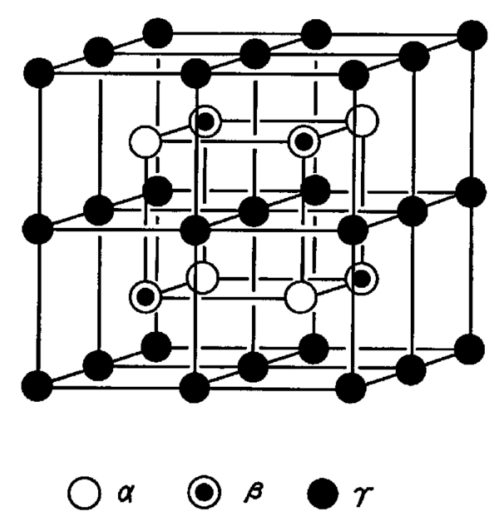

Fig. 1 Unit cell of the Fe-Al system and definition of $\alpha, \beta$ and $\gamma$ sites.

1974 Vol. 15 
Table 1 Occupation numbers of $\mathrm{Fe}$ and $\mathrm{Al}$ atoms on $\alpha, \beta$ and $\gamma$ sites defined in terms of $x$ and $y$.

\begin{tabular}{cccc}
\hline \hline Atoms & $\mathrm{Fe}$ & $\mathrm{Al}$ & $\mathrm{Sum}$ \\
Sites & & $\frac{N}{16}(1+\varepsilon-x+y)$ & $\frac{N}{4}$ \\
\hline$\alpha$ & $\frac{N}{16}(3-\varepsilon+x-y)$ & $\frac{N}{16}(1+\varepsilon+x+y)$ & $\frac{N}{4}$ \\
$\beta$ & $\frac{N}{16}(3-\varepsilon-x-y)$ & $\frac{N}{8}(1+\varepsilon-y)$ & $\frac{N}{2}$ \\
$\gamma$ & $\frac{N}{8}(3-\varepsilon+y)$ & $\frac{N}{4}(1+\varepsilon)$ & $N$ \\
\hline Sum & $\frac{N}{4}(3-\varepsilon)$ & \\
\hline
\end{tabular}

they will change due to the atomic interchange until they reach their equilibrium values.

Taking into account the direct interchange of atoms between the first and the second nearest lattice sites, we describe the ordering of the $\mathrm{Fe}-\mathrm{Al}$ alloys by the following three types of pseudo-chemical reaction:

$$
\begin{aligned}
& \mathrm{Fe}_{\gamma}+\mathrm{Al}_{\alpha} \rightleftarrows \mathrm{Al}_{\gamma}+\mathrm{Fe}_{\alpha}, \\
& \mathrm{Fe}_{\gamma}+\mathrm{Al}_{\beta} \rightleftarrows \mathrm{Al}_{\gamma}+\mathrm{Fe}_{\beta}, \\
& \mathrm{Fe}_{\alpha}+\mathrm{Al}_{\beta} \rightleftarrows \mathrm{Al}_{\alpha}+\mathrm{Fe}_{\beta},
\end{aligned}
$$

where $\mathrm{Fe}_{\alpha}$, for example, signifies $\mathrm{Fe}$ atoms on the sublattice $\alpha$. The left hand side of the above reaction formula represents the favored configuration and the right hand side the unfavored one. The reaction which proceeds from left to right (or in forward direction) corresponds to the disordering, whereas the one from right to left (or in backward direction) to the ordering. Thus if we denote the net rates of the reactions (1-1), (1-2) and (1-3) by $R_{1}, R_{2}$ and $R_{3}$, the rate of change in the degrees of order is obtained by the use of Table 1 as:

$$
\begin{aligned}
& \frac{d x}{d t}=\frac{8}{N}\left(R_{1}-R_{2}-2 R_{3}\right), \\
& \frac{d y}{d t}=-\frac{8}{N}\left(R_{1}+R_{2}\right) .
\end{aligned}
$$

The reaction rates $R_{1}, R_{2}$ and $R_{3}$ are obtained in the following way. Let the potential barriers for the atomic interchange in the reaction (1-1) in the forward and backward directions be denoted by $U_{1}+$ $Q_{1} / 2$ and $U_{1}-Q_{1} / 2$, respectively, and so forth, then the reaction rates are given by:

$$
\begin{aligned}
R_{1}= & 2 N v_{1}\left[p_{\alpha}^{\mathrm{Al}} p_{\gamma}^{\mathrm{Fe}} \exp \left\{-\left(U_{1}+Q_{1} / 2\right) / T\right\}\right. \\
& \left.-p_{\alpha}^{\mathrm{Fe}} p_{\gamma}^{\mathrm{A}} \exp \left\{-\left(U_{1}-Q_{1} / 2\right) / T\right\}\right], \\
R_{2}= & 2 N v_{2}\left[p_{\beta}^{\mathrm{Al}} p_{\gamma}^{\mathrm{Fe}} \exp \left\{-\left(U_{2}+Q_{2} / 2\right) / T\right\}\right. \\
& \left.-p_{\beta}^{\mathrm{Fe}} p_{\gamma}^{\mathrm{A}} \exp \left\{-\left(U_{2}-Q_{2} / 2\right) / T\right\}\right], \\
R_{3}= & \frac{3}{2} N v_{3}\left[p_{\alpha}^{\mathrm{Fe}} p_{\beta}^{\mathrm{Al}} \exp \left\{-\left(U_{3}+Q_{3} / 2\right) / T\right\}\right. \\
& \left.-p_{\alpha}^{\mathrm{A} 1} p_{\beta}^{\mathrm{Fe}} \exp \left\{-\left(U_{3}-Q_{3} / 2\right) / T\right\}\right],
\end{aligned}
$$

where $v_{i}$ is the vibrational frequency of atoms in the $i$-th reaction, and $p_{\alpha}^{\mathrm{Fe}}$, for example, is the probability for an $\alpha$ site to be occupied by an Fe atom, which is easily obtained from Table $1 . Q_{i}$ represents the difference between the energies of a pair of atoms before and after the interchange, and can be obtained with reference to Fig. 1 and Table 1 by considering the atomic interaction between the first and the second nearest neighbors, or

$$
\begin{aligned}
& Q_{1}=V y-W x, \\
& Q_{2}=V y+W x, \\
& Q_{3}=2 W x,
\end{aligned}
$$

where the energy parameters $V$ and $W$ have been defined in our previous paper ${ }^{(15)}$.

Now we assume that between the atomic interchange in the reaction (1-1) and (1-2) the vibrational frequencies and the barrier heights are the same and independent of the degrees of order, or $v_{1}=v_{2}$ and $U_{1}=U_{2}$. From eqs. (2), (3) and (4) we obtain the following simultaneous differential equations which express the rate of change in the degrees of order $x$ and $y$ :

$$
\begin{aligned}
\frac{d x}{d t}= & A(T)[-(3-\varepsilon+x-y)(1+\varepsilon-y) \\
& \times \exp \{(V y-W x) / 2 T\} \\
& +(1+\varepsilon-x+y)(3-\varepsilon+y) \\
& \times \exp \{-(V y-W x) / 2 T\} \\
& +(3-\varepsilon-x-y)(1+\varepsilon-y) \\
& \times \exp \{(V y+W x) / 2 T\} \\
& -(1+\varepsilon+x+y)(3-\varepsilon+y) \\
& \times \exp \{-(V y+W x) / 2 T\}] \\
& +\frac{3}{2} B(T)[(3-\varepsilon-x-y)(1+\varepsilon-x+y) \\
& \times \exp (W x / T) \\
& -(3-\varepsilon+x-y)(1+\varepsilon+x+y) \\
& \times \exp (-W x / T)] \\
\frac{d y}{d t}= & A(T)[(3-\varepsilon+x-y)(1+\varepsilon-y) \\
& \times \exp \{(V y-W x) / 2 T\} \\
& -(1+\varepsilon-x+y)(3-\varepsilon+y) \\
& \times \exp \{-(V y-W x) / 2 T\} \\
& +(3-\varepsilon-x-y)(1+\varepsilon-y) \\
& \times \exp \{(V y+W x) / 2 T\} \\
& -(1+\varepsilon+x+y)(3-\varepsilon+y) \\
& \times \exp \{-(V y+W x) / 2 T\}]
\end{aligned}
$$

where

$$
\begin{aligned}
& A(T)=v_{1} \exp \left(-U_{1} / T\right), \\
& B(T)=v_{3} \exp \left(-U_{3} / T\right) .
\end{aligned}
$$

Here $d x / d t$ is composed of the two terms associated with the interchange of atoms between the first and the second nearest neighbors, whereas $d y / d t$ is made up of only the atomic interchange between the first nearest neighbors.

Setting $d x / d t=0$ and $d y / d t=0$ in eqs. (5-1) and (5-2), we have the equations which determine the equilibrium values of the order parameters, $x_{e}$ and $y_{e}$, at a given temperature: 
$x_{e}=\frac{T}{2 W} \ln \frac{\left(1+\varepsilon+x_{e}+y_{e}\right)\left(3-\varepsilon+x_{e}-y_{e}\right)}{\left(1+\varepsilon-x_{e}+y_{e}\right)\left(3-\varepsilon-x_{e}-y_{e}\right)}$,

$y_{e}=\frac{T}{2 V} \ln \frac{\left(3-\varepsilon+y_{e}\right)^{2}\left(1+\varepsilon+x_{e}+y_{e}\right)\left(1+\varepsilon-x_{e}+y_{e}\right)}{\left(1+\varepsilon-y_{e}\right)^{2}\left(3-\varepsilon+x_{e}-y_{e}\right)\left(3-\varepsilon-x_{e}-y_{e}\right)}$.

These equations are identical with those of our previous investigation ${ }^{(15)}$ for the temperature dependence of the order parameters in the equilibrium state obtained from the $B-W$ approximation under the requirement of minimum free energy.

The critical temperatures $T_{y}$ (disorder $\rightleftarrows \mathrm{B} 2$ ) and $T_{x}$ $\left(\mathrm{B} 2 \rightleftarrows \mathrm{DO}_{3}\right)$ can be obtained easily from eqs. (6-2) and (6-1), respectively.

$$
T_{y}=\frac{V}{8}(3-\varepsilon)(1+\varepsilon),
$$

and

$$
T_{x}=\frac{W}{4}\left(3-\varepsilon-y_{e}\right)\left(1+\varepsilon+y_{e}\right) .
$$

Conversely, according to eqs. (7-1) and (7-2) the interaction energies $V$ and $W$ can be estimated from the experimental values of $T_{y}$ and $T_{x}$, respectively, as will be described later.

In deriving the rate equations (5-1) and (5-2) we have assumed, as an elementary process for the reactions (1-1), (1-2) and (1-3), a direct interchange of atoms between a pair of neighboring lattice sites.

In the actual state the interchange of atoms intermediated by vacancies, or the indirect interchange, may be predominant. However, the indirect interchange model would lead, in effect, to almost the same rate equations as (5-1) and (5-2), with $A(T)$ and $B(T)$ proportional to the vacancy concentration in this case. Thus it seems adequate to investigate the kinetics of ordering in the $\mathrm{Fe}-\mathrm{Al}$ system with the use of our rate equations. Because of the complexity of the right hand sides of eqs. (5-1) and (5-2), these equations cannot be solved rigorously by analytical methods, and in the next subsection we shall obtain approximate solutions numerically, using the method of relaxation time approximation.

\section{Approximate solutions}

In this subsection the differential equations (5-1) and (5-2) are simplified into a form suitable for the analysis of the experimental results. When the right hand sides of eqs. (5-1) and (5-2) are expanded into a Taylor series about $x=x_{e}$ and $y=y_{e}$, and the terms of the second and higher orders are neglected, then the following results are obtained with $X=x_{e}-x$ and $Y=y_{e}-y$ :

$$
\begin{aligned}
& \frac{d X}{d t}=a_{11} X+a_{12} Y, \\
& \frac{d Y}{d t}=a_{21} X+a_{22} Y,
\end{aligned}
$$

where

$$
\begin{aligned}
& a_{11}=A(T)\left[( 1 + \varepsilon - y ) \left[\left\{\frac{W}{2 T}(3-\varepsilon+x-y)-1\right\} \exp \left(\frac{V y-W x}{2 T}\right)\right.\right. \\
& \left.+\left\{\frac{W}{2 T}(3-\varepsilon-x-y)-1\right\} \exp \left(\frac{V y+W x}{2 T}\right)\right] \\
& +(3-\varepsilon+y)\left[\left\{\frac{W}{2 T}(1+\varepsilon-x+y)-1\right\} \exp \left(-\frac{V y-W x}{2 T}\right)\right. \\
& \left.\left.+\left\{\frac{W}{2 T}(1+\varepsilon+x+y)-1\right\} \exp \left(-\frac{V y+W x}{2 T}\right)\right]\right] \prod_{\substack{x=x_{e} \\
y=y_{e}}} \\
& +\frac{3}{2} B(T)\left[\left\{\frac{W}{T}(3-\varepsilon-x-y)(1+\varepsilon-x+y)-2(2-x)\right\} \exp \left(\frac{W x}{T}\right)\right. \\
& \left.+\left\{\frac{W}{T}(3-\varepsilon+x-y)(1+\varepsilon+x+y)-2(2+x)\right\} \exp \left(-\frac{W x}{T}\right)\right]_{\substack{x=x_{e} \\
y=y_{e}}}, \\
& a_{12}=A(T)\left[-\left\{\frac{V}{2 T}(3-\varepsilon+x-y)(1+\varepsilon-y)-(4+x-2 y)\right\} \exp \left(\frac{V y-W x}{2 T}\right)\right. \\
& -\left\{\frac{V}{2 T}(1+\varepsilon-x+y)(3-\varepsilon+y)-(4-x+2 y)\right\} \exp \left(-\frac{V y-W x}{2 T}\right) \\
& +\left\{\frac{V}{2 T}(3-\varepsilon-x-y)(1+\varepsilon-y)-(4-x-2 y)\right\} \exp \left(\frac{V y+W x}{2 T}\right) \\
& \left.+\left\{\frac{V}{2 T}(1+\varepsilon+x+y)(3-\varepsilon+y)-(4+x+2 y)\right\} \exp \left(-\frac{V y+W x}{2 T}\right)\right]_{\substack{x=x_{e} \\
y=y_{e}}} \\
& +3 B(T)(1-\varepsilon-y)\left[\exp \left(\frac{W x}{T}\right)-\exp \left(-\frac{W x}{T}\right)\right]_{\substack{x=x_{e} \\
y=y_{e}}} \\
& a_{21}=A(T) \llbracket(1+\varepsilon-y)\left[-\left\{\frac{W}{2 T}(3-\varepsilon+x-y)-1\right\} \exp \left(\frac{V y-W x}{2 T}\right)\right. \\
& \left.+\left\{\frac{W}{2 T}(3-\varepsilon-x-y)-1\right\} \exp \left(\frac{V y+W x}{2 T}\right)\right]
\end{aligned}
$$




$$
\begin{aligned}
+ & (3-\varepsilon+y)\left[-\left\{\frac{W}{2 T}(1+\varepsilon-x+y)-1\right\} \exp \left(-\frac{V y-W x}{2 T}\right)\right. \\
& \left.\left.\left.+\left\{\frac{W}{2 T}(1+\varepsilon+x+y)-1\right\} \exp \left(-\frac{V y+W x}{2 T}\right)\right]\right]\right]_{y=x_{e}}, \\
a_{22}= & A(T)\left[\left\{\frac{V}{2 T}(3-\varepsilon+x-y)(1+\varepsilon-y)-(4+x-2 y)\right\} \exp \left(\frac{V y-W x}{2 T}\right)\right. \\
& +\left\{\frac{V}{2 T}(1+\varepsilon-x+y)(3-\varepsilon+y)-(4-x+2 y)\right\} \exp \left(-\frac{V y-W x}{2 T}\right) \\
& +\left\{\frac{V}{2 T}(3-\varepsilon-x-y)(1+\varepsilon-y)-(4-x-2 y)\right\} \exp \left(\frac{V y+W x}{2 T}\right) \\
& \left.+\left\{\frac{V}{2 T}(1+\varepsilon+x+y)(3-\varepsilon+y)-(4+x+2 y)\right\} \exp \left(-\frac{V y+W x}{2 T}\right)\right]_{\substack{x=x_{e} \\
y=y_{e}}} .
\end{aligned}
$$

The linear differential equations (8-1) and (8-2) can be easily solved for $X$ and $Y$, and the solutions are given by

$$
X=\frac{1}{C_{1}-C_{2}}\left(F_{1}(t)-F_{2}(t)\right),
$$

and

$$
Y=\frac{1}{C_{1}-C_{2}}\left(C_{1} F_{1}(t)-C_{2} F_{2}(t)\right)
$$

where

$$
\begin{gathered}
F_{1}(t)=\left\{y_{e}-y_{0}-C_{2}\left(x_{e}-x_{0}\right)\right\} \exp \left(\lambda_{1} t\right), \\
F_{2}(t)=\left\{y_{e}-y_{0}-C_{1}\left(x_{e}-x_{0}\right)\right\} \exp \left(\lambda_{2} t\right), \\
\lambda_{1}, \lambda_{2}=\frac{1}{2}\left\{a_{11}+a_{22} \pm \sqrt{\left(a_{11}-a_{22}\right)^{2}+4 a_{12} a_{21}}\right\}, \\
C_{1}, C_{2}=\frac{1}{2 a_{12}}\left\{a_{22}-a_{11} \pm \sqrt{\left(a_{11}-a_{22}\right)^{2}+4 a_{12} a_{21}}\right\},
\end{gathered}
$$

and $x_{0}$ and $y_{0}$ denote the initial values for $x$ and $y$, respectively. In the above approach the assumption is made that the values of $X$ and $Y$ is not large. This restriction excludes the application of the above approximate equations to the transition from the completely disordered state to B2 ordered one, or from $\mathrm{B} 2$ to $\mathrm{DO}_{3}$. When the differences between the initial and the final values for $x$ and $y$ are small, the kinetic behaviors of ordering or disordering are investigated by evaluating the expressions (9-1) and (9-2).

\section{Experimental Procedure}

Figure 2 shows the phase diagram for the $\mathrm{Fe}-\mathrm{Al}$ system as proposed by our previous analysis by X-ray diffraction $^{(16)}$. In the neighborhood of 25 at $\% \mathrm{Al}$ there are two-phase regions $\alpha+\mathrm{B} 2$ and $\alpha+\mathrm{DO}_{3}$ in addition to the single-phase regions $\alpha, \mathrm{B} 2$ and $\mathrm{DO}_{3}$. The kinetic equations which have been set up in section II are based on the assumption of homogeneous ordering. In order to compare the calculated results with the experimental ones the specimens for the experiment are required to have such a composition that there are no two-phase fields in the whole temperature range of the specimens. In addition, they should be heat-treated so that the ordering proceeds as homogeneous as possible.

The specimens were prepared from a single ingot, which was made up of $99.95 \%$ electrolytic iron and $99.99 \%$ aluminum by induction melting and casting into steel mold. After homogenization in vacuum at $900^{\circ} \mathrm{C}$ for $24 \mathrm{hr}$, the ingot was filed into powder finer than 200 mesh for the X-ray experiment. The powder was sealed in evacuated silica tubes, annealed at $850^{\circ} \mathrm{C}$ for $6 \mathrm{hr}$ to remove the effect of cold work, and then underwent either one of the following heat treatments in order to examine the process of homogeneous ordering:

(1) A part of the powdered specimen was slowly cooled from $850^{\circ} \mathrm{C}$ (disordered phase) to an appropriate temperature at a rate of $1^{\circ} \mathrm{C} / \mathrm{min}$, held at that temperature for $2 \mathrm{hr}$ and then quenched into iced brine. Similarly an adequate step cooling was applied to the second, third and other parts, which were then quenched.

(2) Some parts of the specimen were quenched from $526^{\circ} \mathrm{C}\left(\mathrm{DO}_{3}\right.$ phase) after the heat treatment (1), subsequently annealed at $380^{\circ} \mathrm{C}$ or $363^{\circ} \mathrm{C}$ and then quenched.

The specimens thus prepared were in turn set in JDX-7D type diffractometer, and the intensities of reflections of $\mathrm{CoK} \alpha$ X-rays were measured at room

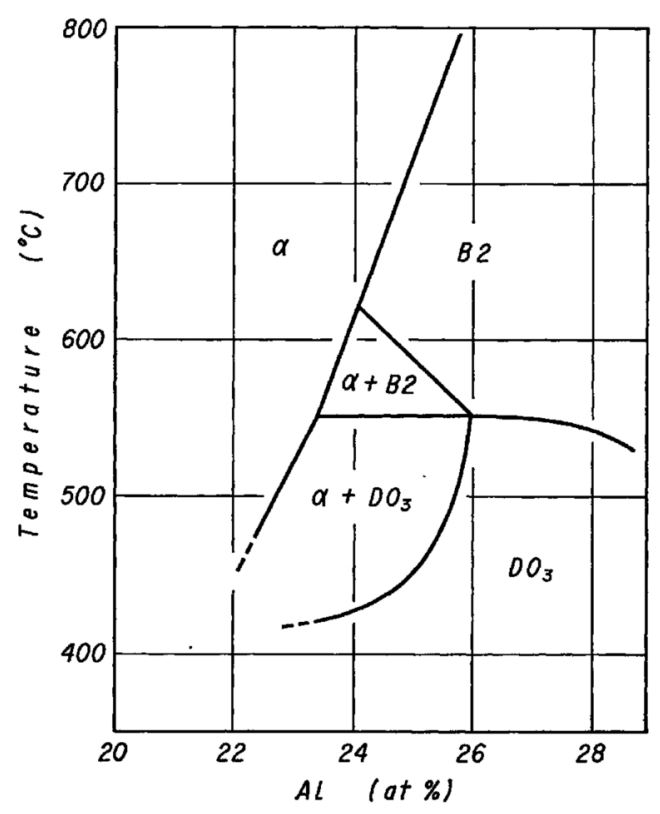

Fig. 2 Phase diagram of the $\mathrm{Fe}-\mathrm{Al}$ system proposed by our previous study ${ }^{(16)}$. 
temperature. The degrees of order $x$ and $y$ were obtained from the integrated intensities of various reflections. The practical procedure for obtaining the degrees of order has been already described in our previous paper ${ }^{(16)}$.

Chemical analysis showed that the specimens contained 26.0 at $\% \mathrm{Al}$.

\section{Experimental Results and Theoretical Interpretation}

In this section we shall report our experimental results of X-ray diffraction and the theoretical analysis of the data using our approximate equations (9-1) and (9-2). Before going into our main argument, let us at first examine the number of unknown parameters in the equations. We can regard the interaction energies $V$ and $W$, which appear in the expansion coefficients $a_{i j}$, as known from the beginning. Referring to Fig. 2 and eq. (7) we can estimate their values as $V=2800^{\circ} \mathrm{K}$ and $W=825^{\circ} \mathrm{K}$ for the alloy with 26.0 at $\% \mathrm{Al}(\varepsilon=$ 0.04). If the initial and final values for $x$ and $y$, or $x_{0}, y_{0}, x_{e}$ and $y_{e}$, are given only the two parameters $A(T)$ and $B(T)$ remain unknown.

The isothermal change in the degrees of order $x$ and $y$ in the alloys heat-treated by (2) is shown in Fig. 3. The value of $y$ increases abruptly with annealing time and reaches its stationary value within a few minutes, while that of $x$ exhibits a further increase showing the homogeneous ordering of the $\mathrm{DO}_{3}$ structure even after the saturation of $y$, and the increase lasts for about $10 \mathrm{~min}$. As an initial approach to the analysis we first make an assumption that the ordering proceeds by the interchange of atoms between the first nearest neighbors only. This assumption implies the neglect of the term with $B(T)$ in eq. (8-1). The numerical calculation has been performed with various values for the parameter $A(T)$, and with the observed values for the initial and final values of $x$ and $y$. The results are illustrated in Fig. 3(a).

The calculated values of $x$ and $y$ show a large dependence on the variation of $A(T)$. Since the B2 ordering, which is characterized by the degree of order $y$, is caused by the atomic interchange between the first nearest neighbors, the value for the parameter $A(T)$ can be determined so that the calculated value of $y$ reproduces the observed one. The curve (c) in Fig. $3(\mathrm{a})$ is the best fit for the experimental data on $380^{\circ} \mathrm{C}$ annealing, but the values for $x$ do not represent the observed data satisfactorily. It is obvious that the disagreement of $x$ values is caused by the neglect of the second nearest interchange of atoms. Now the latter effect is introduced by assuming a finite value for the parameter $B(T)$ and the calculation is repeated. The results are shown in Fig. 3(b). The value of $y$ scarcely changes in spite of the variation of $B(T)$, while $x$ changes remarkably.

The final calculated results for 380 and $363^{\circ} \mathrm{C}$ annealing are shown together with the observed data in Fig. 4, where the close agreement between the theoretical and experimental results is seen. From the

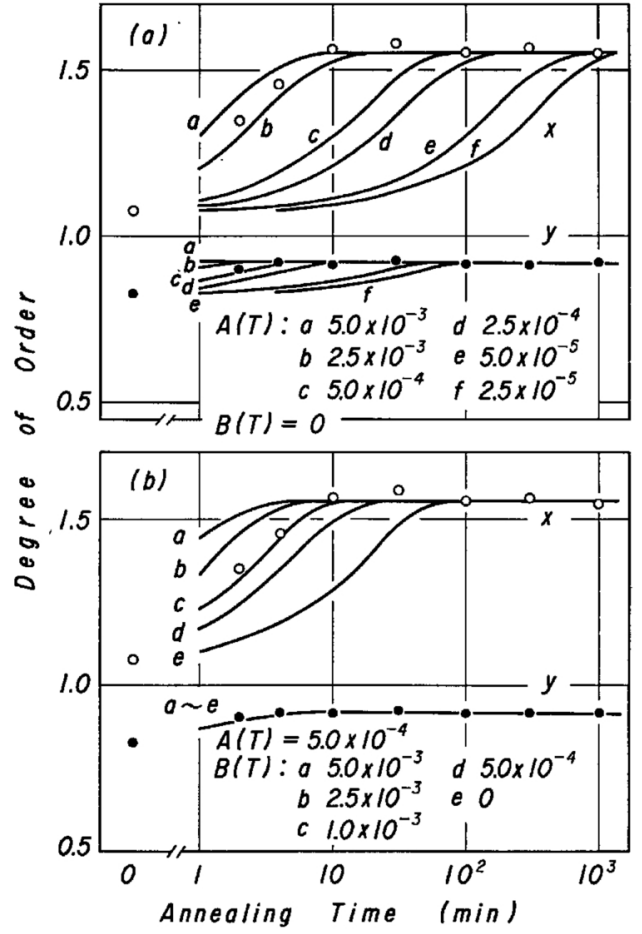

Fig. 3 Comparison of calculated curves for degrees of order $x$ and $y$ with corresponding experimental data (open circles and dots) obtained in the isothermal annealing at $380^{\circ} \mathrm{C}$. Calculated results by considering the atomic interchange between the first nearest neighbors only (a), and between the first and the second nearest neighbors (b).

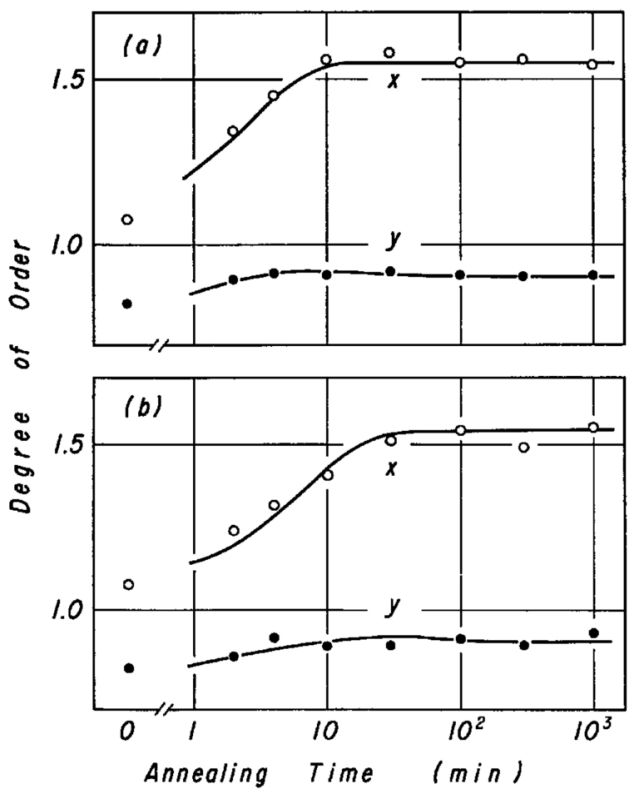

Fig. 4 Isothermal change in degrees of order $x$ and $y$ obtained by the experiment (open circles and dots) and the theoretical values (solid curves) at $380^{\circ} \mathrm{C}$ (a) and $363^{\circ} \mathrm{C}(\mathrm{b})$.

values obtained for the parameters $A(T)$ and $B(T)$ the following values are chosen for the heights of potential barrier and the vibrational frequencies.

$$
\begin{array}{ll}
U_{1}=23000^{\circ} \mathrm{K}, & v_{1}=1 \times 10^{12} / \mathrm{sec}, \\
U_{3}=23000^{\circ} \mathrm{K}, & v_{3}=2 \times 10^{12} / \mathrm{sec} .
\end{array}
$$


It should be emphasized that the interchange of atoms between the first nearest neighbors only does not account for the experimental results satisfactorily, and one has to consider the contribution of the second nearest interchange of atoms.

Having thus determined the parameters in the fundamental equations (5-1) and (5-2), we now proceed to analyze the experimental results on the quenched specimens heat-treated by (1). The variation in the degrees of order $x$ and $y$ with quenching temperature $T_{q}$ is shown in Fig. 5. The value of $x$ is zero for $T_{q}>T_{x}$, whereas $y$ takes a roughly constant and large value there. The large value of $y$ means the progress of $\mathrm{B} 2$ type ordering during the quench ${ }^{(17)(18)}$. Below $T_{x} x$ increases continually with decreasing $T_{q}$, but the value of $y$ increases only slightly. In Fig. 5 the result of calculation with our eqs. (5-1) and (5-2) is shown for the values of $x$ and $y$ as functions of the quenching temperature $T_{q}$ with the quenching rate of $1000^{\circ} \mathrm{C} / \mathrm{sec}$. Considering the accuracy of the experimental data and the approximate nature of our rate equations the agreement between theory and experiment is satisfactory. It should be noted that the B2 ordering takes place so rapidly that the conventional method of quenching does not prevent the ordering. Even if we assume a drastic quenching rate as high as $50000^{\circ} \mathrm{C} / \mathrm{sec}$, the calculation shows the $\mathrm{B} 2$ ordering during the quench. This tendency is also consistent with the experimental result obtained by Swann et al. ${ }^{(18)}$ by electron microscopy.

Figure 6 shows the contour lines of $d x / d t$ and $d y / d t$ in the $x y$-plane as given by eqs. (5-1) and (5-2) in the case of annealing at $380^{\circ} \mathrm{C}$. In the figure the trajectory of ordering when the alloy is annealed at that temperature after it was quenched from $526^{\circ} \mathrm{C}$ is also shown. As is seen in the figure, $d y / d t$ decreases with

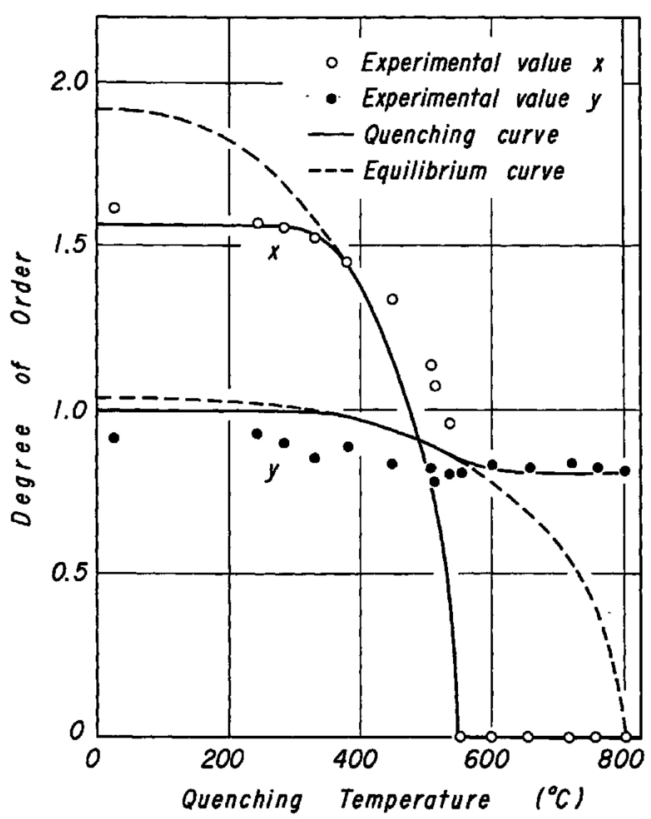

Fig. 5 Temperature dependence of degrees of order $x$ and $y$ obtained by the experiment (open circles and dots) and the theoretical result (solid and dashed curves).

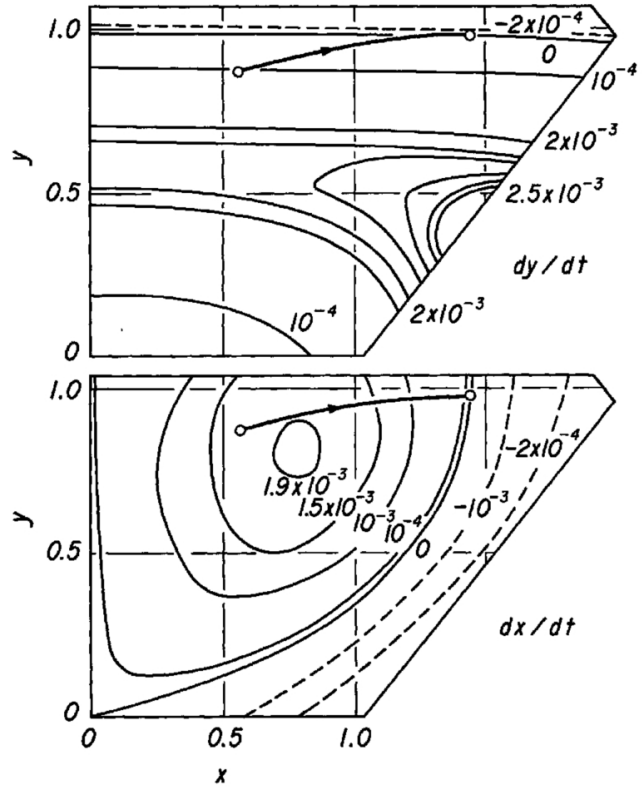

Fig. 6 Contour lines of $d x / d t$ and $d y / d t$, and the trajectory of ordering on $380^{\circ} \mathrm{C}$ annealing.

annealing time, reduces to the minimum negative value after passing through zero, and gradually approaches its equilibrium value. Namely, $y$ does not increase monotonously but oscillates due to its correlation with $x$. The oscillatory change of $y$, however, is small and cannot be detected experimentally within the accuracy of the present experiment.

\section{Conclusion}

The kinetic equations which describe the ordering in the $\mathrm{Fe}-\mathrm{Al}$ alloys with two types of superstructures B2 and $\mathrm{DO}_{3}$ have been obtained by considering the direct interchange of atoms between the first and the second nearest neighbors. The approximate solutions of these equations are shown to fit well the experimental results of X-ray diffraction, and it is shown that the analysis of the results using a linear approximation is useful.

The heights of potential barriers and the vibrational frequencies for the first and the second nearest interchange of atoms have been evaluated, and it has been shown that the ordering of $\mathrm{DO}_{3}$ superstructure is influenced substantially by the atomic interchange between the second nearest neighbors. The B2 ordering sets in so rapidly at high temperatures that it proceeds even in the course of quenching from the disordered state.

The oscillatory change of the degree of order in B2 structure is expected from the calculation of $y$ in the isothermal annealing. This might be an essential feature of ordering in the $\mathrm{Fe}-\mathrm{Al}$ system, but we must wait for the experimental confirmation in future.

\section{REFERENCES}

(1) W. L. Bragg and E. J. Williams: Proc. Roy. Soc., A145 (1934), 699. 
(2) Y. Takagi and T. Oguchi: Bull. Tokyo Inst. Tech., B3 (1950), 211.

(3) G. J. Dienes: Acta Met., 3 (1955), 549.

(4) S. Iida: J. Phys. Soc. Japan, 10 (1955), 769.

(5) G. H. Vineyard: Phys. Rev., 102 (1956), 981.

(6) A. S. Nowick and L. R. Weisberg: Acta Met., 6 (1958), 260.

(7) H. Matsuda, H. Kuroki and T. Eguchi: Trans. JIM, 12 (1971), 390.

(8) H. J. McQueen and G. C. Kuczynski: Trans. Met. Soc. AIME, 215 (1959), 619.

(9) R. Feder and R. W. Cahn: Phil. Mag., 5 (1960), 343.

(10) J. Bransky and P. S. Rudmann: Trans. ASM, 55 (1962), 335.

(11) R. G. Davies: Trans. Met. Soc. AIME, 230 (1964), 903.
(12) Ya. P. Selisskiy: Fiz. Metal. Metalloved., 21 (1966), 894; 22 (1966), 87.

(13) K. Oki, M. Hasaka and T. Eguchi: Trans. JIM, 14 (1973), 8.

(14) A. J. Bradley and A. H. Jay: Proc. Roy. Soc., A136 (1932), 210; J. Iron Steel Inst., 125 (1932), 339.

(15) T. Eguchi, H. Matsuda, K. Oki, S. Kiyoto and K. Yasutake: Trans. JIM, 8 (1967), 174.

(16) K. Oki, M. Hasaka and T. Eguchi: Japan. J. Appl. Phys., 12 (1973), 1522.

(17) M. J. Marcinkowski and N. Brown: J. Appl. Phys., 33 (1962), 537.

(18) P. R. Swann, W. R. Duff and R. M. Fisher: Trans. Met. Soc. AIME, 245 (1969), 851; Met. Trans., 3 (1972), 409. 